\title{
A NOTE ON CONVERGENCE IN AREA
}

\author{
R. G. HELSELL
}

Let $T_{n}: x=x_{n}(u, v), y=y_{n}(u, v), z=z_{n}(u, v), n=0,1, \cdots$, be a sequence of continuous transformations from the unit square $S$ : $0 \leqq u, v \leqq 1$, into $E_{3}$, Euclidean 3-space. For each point $p$ on the unit sphere $U: x^{2}+y^{2}+z^{2}=1, E_{2}(p)$ will denote the plane through the origin which is perpendicular to the radius joining the origin and $p$; $T_{p}$ will denote the transformation which projects $E_{3}$ perpendicularly onto $E_{2}(p)$. Then $T_{p} T_{n}$ is a sequence of continuous transformations from $S$ into $E_{2}(p)$. Assume that $T_{n}, n=1,2, \cdots$, converges uniformly on $S$ to $T_{0}$ and that the Lebesgue area $A\left(T_{n}\right)$ of the $F$-surface (see $[1, \text { II.3.44 }]^{1}$ ) determined by the transformation $T_{n}$ is finite for $n=0,1, \cdots$. The purpose of the present note is to call attention to the fact that recent results of Helsel [2] and Rado [2, 3] imply the following theorem:

THEOREM. Under the assumptions stated above, a necessary and suffcient condition for $A\left(T_{n}\right) \rightarrow A\left(T_{0}\right)$ is that $A\left(T_{p} T_{n}\right) \rightarrow A\left(T_{p} T_{0}\right)$ for every position of the point $p$ on $U$.

This theorem is the analogue for the Lebesgue area of a new result on convergence in length established by Ayer and Radó [4].

Proof. The necessity of the condition has been proved by Rad6 [3]. To establish the sufficiency of the condition, use will be made of the following formula for the Lebesgue area of the $F$-surface determined by $T_{n}$ (see [2]):

$$
A\left(T_{n}\right)=2\left[\frac{1}{4 \pi} \iint_{U} A\left(T_{p} T_{n}\right) d \sigma\right]
$$

$d \sigma$ being the area element on $U$. In [2] it is shown that the Lebesgue area $A\left(T_{n}\right)$ is equal to twice the integral mean value over $U$ of the lower area $a\left(T_{p} T_{n}\right)$ of the flat $F$-surface determined by the transformation $T_{p} T_{n}$; however, the Lebesgue area $A\left(T_{p} T_{n}\right)$ is equal to the lower area $a\left(T_{p} T_{n}\right)$ (see [5], [1, V.2.58], and [6]) so (1) follows. The assumption that $A\left(T_{p} T_{n}\right) \rightarrow A\left(T_{p} T_{0}\right)$ for every point $p$ on $U$ implies, in view of (1), that $A\left(T_{n}\right) \rightarrow A\left(T_{0}\right)$ if termwise integration of the sequence $A\left(T_{p} T_{n}\right)$ is permissible. To show that such is the case, a uniform bound for the functions $A\left(T_{p} T_{n}\right)$ will be displayed. First ob-

Received by the editors September 5, 1948 and, in revised form, October 4, 1948.

1 Numbers in brackets refer to the bibliography at the end of the paper. 
serve that

$$
A\left(T_{p} T_{n}\right) \leqq A\left(T_{n}\right) .
$$

Also, by a fundamental result of Cesari [5],

$$
A\left(T_{n}\right) \leqq A\left(T_{p_{1}} T_{n}\right)+A\left(T_{p_{2}} T_{n}\right)+A\left(T_{p_{3}} T_{n}\right),
$$

where $p_{1}, p_{2}, p_{3}$ are any three points on $U$ such that the radii joining these points to the origin are mutually perpendicular. Regarding $p_{1}, p_{2}, p_{3}$ as fixed, the relations $A\left(T_{p_{i}} T_{n}\right) \rightarrow A\left(T_{p_{i}} T_{0}\right), i=1,2,3$, imply the existence of a constant $K$ such that $A\left(T_{p_{i}} T_{n}\right) \leqq K$ for $n=0,1, \cdots$ and $i=1,2,3$. Hence, from (2) and (3), $A\left(T_{p} T_{n}\right) \leqq 3 K$ for all $p \in U$ and $n=0,1, \cdots$, which completes the proof of the sufficiency.

\section{BIBLIOGRAPHY}

1. T. Rad6, Length and area, Amer. Math. Soc. Colloquium Publications, vol. 30 1948.

2. R. G. Helsel and T. Rad6, The Cauchy area of a Frechet surface, Duke Math. J. vol. 15 (1948) pp. 159-167.

3. T. Rad6, On convergence in area, Duke Math. J. vol. 16 (1949) pp. 61-71.

4. M. C. Ayer and T. Rad6, $A$ note on convergence in length, Bull. Amer. Math. Soc. vol. 54 (1948) pp. 533-539.

5. L. Cesari, Caratterizzazione analitica delle superficie continue di area finita secondo Lebesgue, Annali della Reale Scuola Normale Superiore di Pisa (2) vol. 10 (1941) pp. 253-294 and vol. 11 (1942) pp. 1-42.

The OHio State University 